\title{
Computers
} \& Structures

\section{Fuzzy optimization of structures by the two-phase method}

\author{
L.M.C. Simões \\ Department of Civil Engineering, University of Coimbra, 3030 Coimbra, Portugal
}

Received 8 February 2000; accepted 30 March 2001

\begin{abstract}
This work presents the two-phase method for fuzzy optimization of structures. In the first phase the fuzzy solution is obtained by using the level cuts method and in the second phase the crisp solution, which maximizes the membership function of fuzzy decision making, is found by using the bound search method. Illustrative numerical examples involving skeletal structures and reinforced concrete slabs are solved. (c) 2001 Civil-Comp Ltd. and Elsevier Science Ltd. All rights reserved.
\end{abstract}

Keywords: Structural optimization; Fuzzy optimization; Frames; Slabs

\section{Introduction}

In design and optimization problems material constants, loading and structure geometry are usually considered as given data, but in real world assumed values do not correspond with actual ones. Therefore there may be differences between nominal and real geometry, materials may behave in a different way than the assumed one, constant loading may actually vary during the structure lifetime. All of this is accounted by safety factors, which amplify load magnitude, or reduce material strength, leading in general to over-conservative structures.

As an alternative to safety factors one may try to describe the uncertain data and use this information during the optimization, which in general leads to better results in term of optimal design [1,2].

A probabilistic description is nowadays common, and very simple up to very sophisticated PDFs can be used to describe uncertain parameters. However these procedures face serious difficulties when being implemented in engineering applications. This leads to a nonprobabilistic description of uncertainty, in particular the fuzzy set based analysis and the worst condition produced on the constraints by a certain load condition also termed anti-optimization. The latter fixes bounds for the uncertain variables instead of defining probability functions needing much less information than the probabilistic approach [3]. The two-phase method for fuzzy optimization of structures, based on the fuzzy-set method first proposed by Zadeh in Ref. [4], is proposed in this work. In the first phase, the sequential fuzzy solution is obtained by using the level cuts method, in which a fuzzy optimization problem is transformed into a series of ordinary optimization problems using different $\alpha$-level cuts in the fuzzy constraints so as to determine a fuzzy optimization domain in the design space. This procedure has been suggested in Refs. [5,6]. In the second phase, the particular crisp solution is obtained by the bound search method, in which having obtained the supremum and the infimum of the sequential fuzzy solution the particular optimum level $\alpha^{*}$ is found using the bound search so as to provide a crisp optimization solution in the design space. This method differs from that suggested in Ref. [6] on the basis of an alternative interpretation of the Belman-Zadeh optimality criteria [7] which does not require the use of an artificial fuzzy objective. Finally, the two-phase method is illustrated by numerical examples of the fuzzy plastic limit design of frames and slabs.

\section{Fuzziness in structural design}

A fuzzy information problem of structures can be stated as: Find the design vector $\underline{x}$ which minimizes the 
objective function $W(\underline{x})$ subject to fuzzy constraints on performance characteristics and dimensions. This is a fuzzy mathematical programming problem, which can be expressed in a standard form as follows (MP1):

$$
\begin{aligned}
& \min W(\underline{x}) \\
& \text { st : } g_{j}(\underline{x}) \lesssim b_{j}^{\mathrm{U}}, \quad j=1,2, \ldots, m-1 \\
& g_{j}(\underline{x}) \gtrsim b_{j}^{\mathrm{L}}, \quad j=m, \ldots, p
\end{aligned}
$$

where the wave symbols indicate that the constraints contain fuzzy information, and $b_{j}^{\mathrm{U}}, b_{j}^{\mathrm{L}}$ are allowable upper and lower limits of the $j$ th constraint respectively.

The membership function $\mu_{j}(\underline{x})$ of the fuzzy allowable interval may be characterized as shown in Fig. 1. where $b_{j}^{\mathrm{L}}$ and $b_{j}^{\mathrm{U}}$ are respectively the lower and upper limits of the allowable interval for the highest (most rigorous) design level. These may even be more strict than the specifications codes (to be chosen by engineering requirements). $d_{j}^{\mathrm{L}}$ and $d_{j}^{\mathrm{U}}$ are lengths of transition stages, namely the permissible deviations or tolerances for the lower and upper limits. Thus, MP1 can be rewritten in the following form (MP2):

$$
\begin{array}{ll}
\min \quad W(\underline{x}) & \\
\text { st }: & g_{j}(\underline{x}) \leqslant b_{j}^{\mathrm{U}}+d_{j}^{\mathrm{U}}, \quad j=1,2, \ldots, m-1 \\
g_{j}(\underline{x}) \geqslant b_{j}^{\mathrm{L}}-d_{j}^{\mathrm{L}}, \quad j=m, \ldots, p
\end{array}
$$

A proper function may be selected for the transition stage curves of the membership function in the light of the character of the physical variable $g_{j}$. Usually, inclined straight lines may be adopted for simplification.

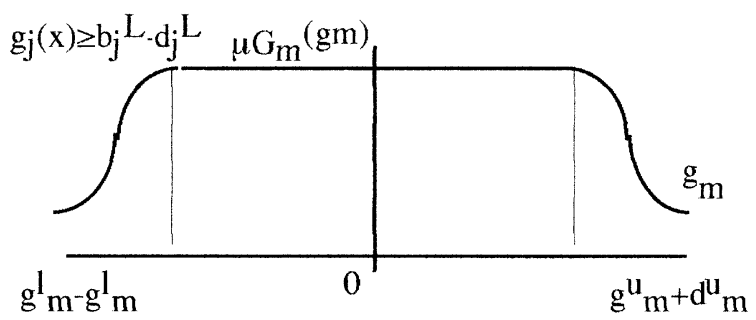

Fig. 1. Nonlinear membership function.

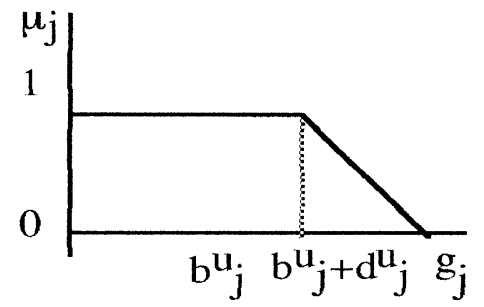

Membership degree $\mu_{j}(\underline{x})$ may be defined as "degree of satisfaction" of the fuzzy constraint. When $\mu_{j}(\underline{x})=1$, the constraint is satisfied completely: when $\mu_{j}(\underline{x})=0$ it is not and when its value lies between 0 and 1 , the constraint is satisfied to the relevant degree.

Some fuzzy information may also be contained in the objective function $W(\underline{x})$. For example, in objective function

$W(\underline{x})=C(\underline{x})+E(\underline{x})$

$C(\underline{x})$ is the initial fabrication cost of the structure, $E(\underline{x})$ is the expected value of additional expenses during its operation, such as maintenance cost, damage losses under disaster and restoration cost.

\section{Two-phase method}

\subsection{First phase (level cuts method)}

For the sake of simplicity only the method of solving problems with fuzzy constraints is discussed in this work. If the membership function of inclined straight lines may be adopted, as shown in Fig. 2, MP2 can be transformed into a nonfuzzy mathematical programming at $\alpha$-level as follows (MP3):

$$
\begin{array}{ll}
\min \quad W(\underline{x}) & \\
\text { st }: & g_{j}(\underline{x}) \leqslant b_{j}^{\mathrm{U}}+d_{j}^{\mathrm{U}}(1-\alpha), \quad j=1,2, \ldots, m-1 \\
& g_{j}(\underline{x}) \geqslant b_{j}^{\mathrm{L}}-d_{j}^{\mathrm{L}}(1-\alpha), \quad j=m, \ldots, p \\
& \alpha \in[0,1]
\end{array}
$$

This is a parametric mathematical programming in $\alpha \in[0,1]$, which can be solved by means of an algorithm for optimization so as to determine the fuzzy optimization solution $x^{*}(\alpha)$ and $W\left(x^{*}(\alpha)\right)$ with different $\alpha$ values. It is noted that a fuzzy optimization problem may have mixed fuzzy and crisp constraints. In this case we shall accept tolerances only on the realization of fuzzy constraints, but crisp ones will completely satisfied. As shown in Fig. 3, the $W\left(x^{*}(\alpha)\right)$ curve defines a fuzzy solution to the fuzzy optimization problem of structures, which is a monotone increasing function of $\alpha$.

Fig. 2. Linear membership function. 


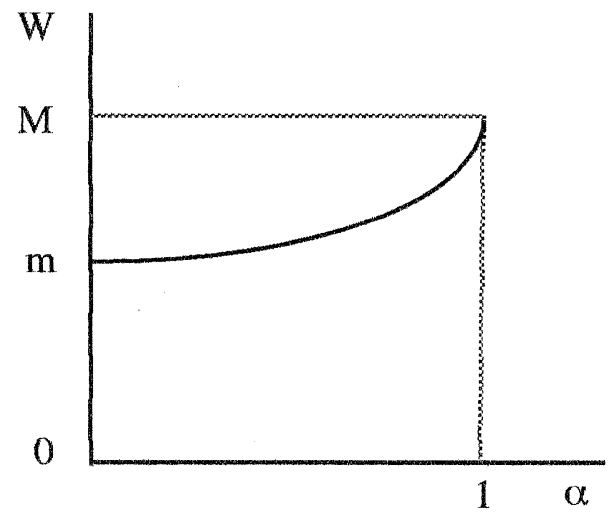

Fig. 3. Fuzzy solution.

\subsection{Second phase (bound search method)}

The Bellman-Zadeh criterion [7] of decision making in a fuzzy environment gives the grade of membership of a decision specified by variables $x$ as

$\mu_{d}(x)=\min _{i} \mu_{i}(x)$

where $i$ ranges over the complete set of constraints. The fuzzy constraints $C$ and the fuzzy goal $G$ in MP3 are defined as fuzzy sets in the space of alternatives, characterized by their membership functions $\mu_{\mathrm{C}}$ and $\mu_{\mathrm{D}}$ respectively. Generally speaking, the fuzzy decision D characterized by its membership function $\mu_{\mathrm{D}}$ may be viewed as the intersection of the fuzzy constraints and the fuzzy goal.

The optimal decision is to select the best alternative from those contained in the fuzzy decision space, which maximizes the membership function of the fuzzy decision, i.e.

$\mu_{\mathrm{D}}\left(x^{*}\right)=\max _{x \varepsilon R} n \mu_{\mathrm{D}}(x)$

In order to illustrate the above principle, consider one fuzzy goal $\mathrm{G}$ with one fuzzy constraint $\mathrm{C}$. The membership functions $\mu_{\mathrm{G}}, \mu_{\mathrm{C}}$ and their intersection $\mu_{\mathrm{D}}$ are plotted for this case in Fig. 4. This figure also shows that the point A represents the optimal decision which has

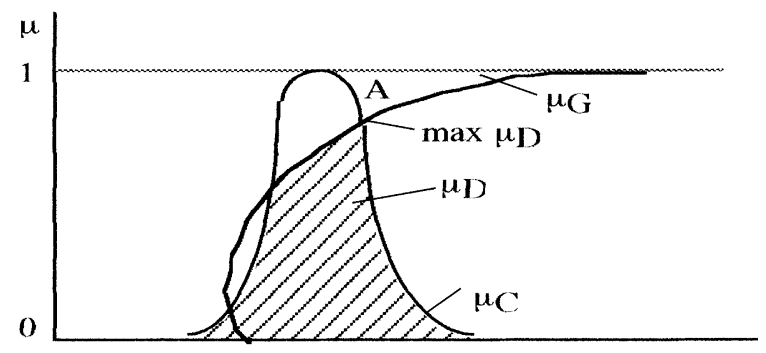

Fig. 4. Fuzzy decision making. the maximum degree of membership in the fuzzy decision set.

From Eq. (6), as demonstrated in Ref. [7], the particular optimum level $\alpha^{*}$ and the optimum point $x^{*}$ are such that:

$\mu_{\mathrm{G}}\left(x^{*}\right)=\max _{x \varepsilon \mathrm{C} \alpha} \mu_{\mathrm{G}}(x)$

where $_{\mathrm{C} \alpha}$ is the $\alpha^{*}$ level cut of the fuzzy constraint set C.

Now it is necessary to establish the fuzzy goal $\mu_{\mathrm{G}}(\underline{x})$ and its upper and lower limits. It can be seen from Fig. 3 that the supremum and the infimum in the sequential fuzzy solution are given by

$M=W\left(x^{*}(1)\right)=\min _{x \varepsilon \mathrm{C}_{1}} W(x)$

$m=W\left(x^{*}(0)\right)=\min _{x \varepsilon \mathrm{C}_{0}} W(x)$

where $C_{1}, C_{0}$ are the level cuts of $\alpha=1$ and 0 of the fuzzy constraint set $\mathrm{C}$.

In the problem of finding $x$ which maximizes the objective function $W(x)$ subject to fuzzy constraints, the fuzzy goal is: $\mu_{\mathrm{G}}(x)=W(x) / \mathrm{M}$. Similarly, in a fuzzy optimization problem of structures to find $x$ which minimizes the objective function $W(x)$ subject to fuzzy constraints, the fuzzy goal can be established as follows:

$\mu_{\mathrm{G}}(x)=m / W(x)$

As expected, this fuzzy goal shows that full membership $\left(\mu_{\mathrm{G}}=1\right)$ is obtained when $W$ reaches its infimum $m$; as $W$ increases $\mu_{\mathrm{G}}$ approaches nonmembership $\left(\mu_{\mathrm{G}}=0\right)$. Clearly, the upper and lower limits of the fuzzy goal are given by:

$\mu_{\mathrm{G}}^{\mathrm{U}}=1$

$\mu_{\mathrm{G}}^{\mathrm{L}}=m / M$

As mentioned in Ref. [8], the optimum level can be derived from Eq. (7) as:

$\alpha^{*}=\mu_{\mathrm{G}}\left(x^{*}\left(\alpha^{*}\right)\right)$

\section{Structural relations}

\subsection{Frames}

For the plastic limit design of a steel frame, the simplest class of problems is when (i) the geometry and topology are fixed; (ii) a single loading state is considered; (iii) the constitutive relations are those of perfect plasticity; (iv) the plasticity is controlled by a single stress resultant. 
The mesh primal LP takes the following form:

$$
\begin{aligned}
& \min z=\left[\begin{array}{ll}
l^{\mathrm{t}} & 0
\end{array}\right]\left[\begin{array}{l}
d \\
p
\end{array}\right] \\
& \text { st : }\left[\begin{array}{cc}
J & -B \\
J & B
\end{array}\right]\left[\begin{array}{l}
d \\
p
\end{array}\right] \geqslant\left[\begin{array}{c}
m_{0} \\
-m_{0}
\end{array}\right] \\
& d \geqslant 0
\end{aligned}
$$

where $d$ are the design variables and $l$ are the lengths of the members associated with the corresponding design variables. The mesh actions (indeterminacies) are denoted by $p$ and $B$ is the mesh static matrix. The matrix $J$ describes the incidence of the design variables with respect to the critical sections and $m_{0}$ is the vector of the particular solution stress resultant at the $i$ th critical section. If the stipulations of the constraint set are fuzzified the problem becomes the parametric linear program:

$$
\begin{aligned}
& \min z=\left[\begin{array}{ll}
l^{\mathrm{t}} & 0
\end{array}\right]\left[\begin{array}{l}
d \\
p
\end{array}\right] \\
& \text { st : }\left[\begin{array}{cc}
J & -B \\
J & B
\end{array}\right]\left[\begin{array}{l}
d \\
p
\end{array}\right] \geqslant\left[\begin{array}{cc}
m_{0} & -d_{0}(1-\alpha) \\
-m_{0} & -d_{0}(1-\alpha)
\end{array}\right] \\
& \quad d \geqslant 0
\end{aligned}
$$

The solution gives a deterministic design $d$ together with a level of acceptability $\alpha$ in the face of fuzziness of the load and plastic moment capacities.

\subsection{Reinforced concrete slabs}

For the class of problems indicated before, the primal LP for plastic limit synthesis in the nodal description using a linearized yield condition incorporating finite elements are:

$$
\begin{aligned}
& \min z=\left[\begin{array}{ll}
c^{\mathrm{t}} & 0
\end{array}\right]\left[\begin{array}{l}
d \\
m
\end{array}\right] \\
& \mathrm{st}:\left[\begin{array}{cc}
J & -U^{\mathrm{t}} \\
0 & -E^{\mathrm{t}} \\
I & 0
\end{array}\right]\left[\begin{array}{l}
d \\
m
\end{array}\right] \geqslant\left[\begin{array}{l}
0 \\
r_{0} \\
\underline{d}
\end{array}\right]
\end{aligned}
$$

The program seeks the "best" yield-line pattern that can be attained when the yield lines are confined to the F.E. boundaries. As for skeletal structure problems, the constraints represent the static admissibility. For any selected F.E. pattern, the plastic flexural deformations are confined to the element boundaries whilst the interiors of the elements remain undeformed plastically. Such deformations correspond to the collapse mode. This method automates the yield-line search within the selected F.E. system.

The vector $c$ is defined by,

$$
c^{\mathrm{t}}=\left[\begin{array}{llll}
a^{+\mathrm{t}} & J^{+} & a^{-\mathrm{t}} & J^{-}
\end{array}\right]
$$

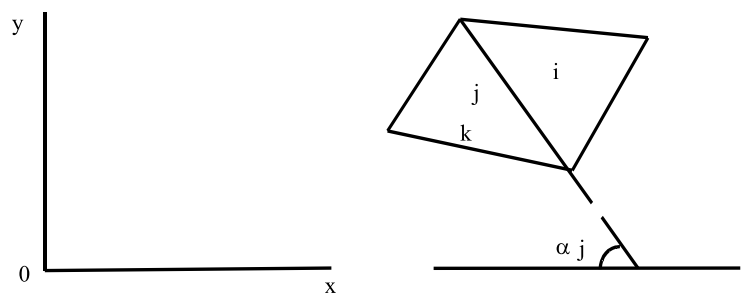

Fig. 5. Yield lines.

where $J^{+}$and $J^{-}$are the incidence matrices relating the plastic moment capacity of the finite elements $m^{* x}, m^{* y}$ to the design variables $d$ for positive and negative bending moments respectively. $a^{+}$(or $a^{-}$) are vectors of known constants $S_{i} /\left(f_{y} h_{i}^{x}\right)$ and $S_{i} /\left(f_{y} h_{i}^{y}\right)$ for positive (or negative) bending where $S_{i}$ is the area of element $i, f_{y}$ the yield stress of the reinforcement and $h_{i}^{x}, h_{i}^{y}$ are the lever arms:

$J=\left[\begin{array}{ll}J_{*}^{+} & \\ & J_{*}^{-}\end{array}\right]$

where $J_{*}^{+}=\left[\begin{array}{ll}A & B\end{array}\right] J^{+}$and $J_{*}^{-}=\left[\begin{array}{ll}A & B\end{array}\right] J^{-}$. The elements of matrices $A$ and $B$ are $l_{j} \sin ^{2} \theta_{j}$ and $l_{j} \cos ^{2} \theta_{j}$, respectively where $\theta_{j}$ is the angle between the interelement side of length $l_{j}$ and the $x$-axis (Fig. 5).

Matrix $U$ is defined by,

$U=\left[\begin{array}{llll}I & I & -I & -I\end{array}\right]$

where $I$ is the identity matrix.

The matrix $I$ relates the vector of applied nodal forces $r_{0}$ to the vector of total bending moments $\mathrm{m}$ across the FE boundaries in the static equation:

$r_{0}=E^{\mathrm{t}} m$

$\underline{d}$ is the lower bound on the design variables $d$. The elements of the matrices $J^{+}$and $J^{-}$are fixed a priori by the designer while the elements of the matrices $E, A$ and $B$ are determined from the slab geometry.

In general, the overall geometry of the slab and the reinforcement strength are known deterministically, whilst the magnitudes of the applied loading and the lower bound on $d$ can only be stated imprecisely. Thus the primal LP becomes one in which the stipulations are imprecise and can be readily tackled by the techniques of FLP. At the $i$ th node, the deterministic equilibrium conditions can be written as

(i) $\sum_{j} e_{j i} m_{j} \leqslant r_{0 i}$

(ii) $\sum_{j} e_{j i} m_{j} \geqslant r_{0 i}$

The vertical nodal forces $r_{0}$ are now considered to be fuzzy numbers. By combining the above conditions with the fuzziness indicated in Fig. 6, the following inequalities result: 


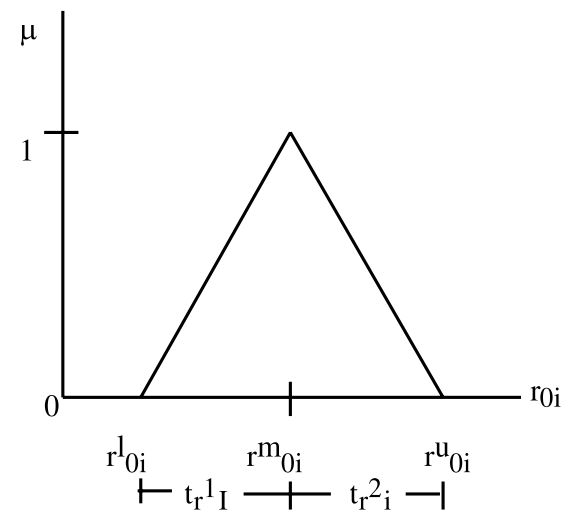

Fig. 6. Membership function for the applied nodal force.

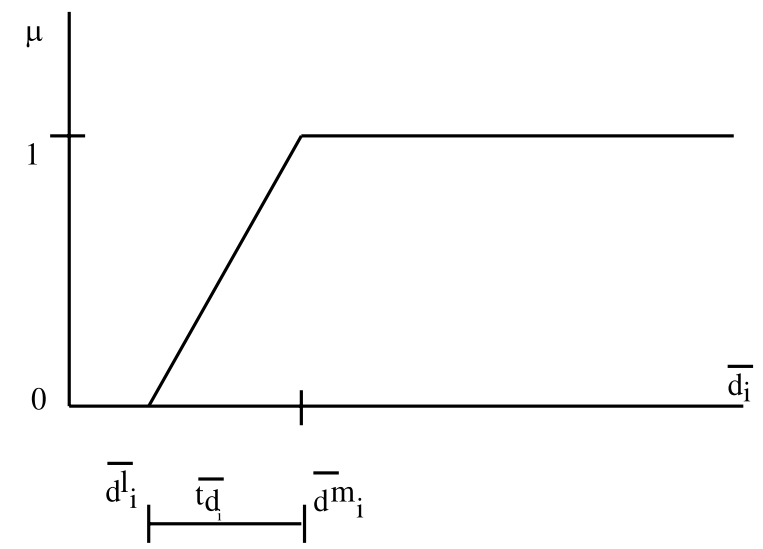

Fig. 7. Membership function for the lower bound on design variable $d_{i}$.

(i) $\sum_{j} e_{j i} m_{j} \leqslant r_{0 i}+t_{r i}^{2}(1-\alpha)$

(ii) $\sum_{j} e_{j i} m_{j} \geqslant r_{0 i}-t_{r i}^{1}(1-\alpha)$

Similarly, the lower bounds on the design variables may be fuzzified as indicated in Fig. 7.

The designer might accept a design variable of value $\underline{d}_{i}^{\mathrm{L}}$ but would much prefer it to be equal to or greater than $\underline{d}_{i}$ $d_{i} \geqslant d_{i}-t_{\mathrm{d} i}(1-\alpha)$

\section{Numerical examples}

\subsection{Prismatic beam}

The fixed-end beam of Fig. 8 is to be designed so it resists the single applied loading without collapsing plastically. The design process consists of fixing the cross-sectional properties and the single design variable is the plastic moment capacity of beam $d$. The designer

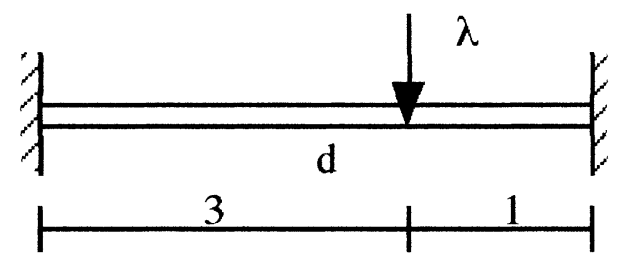

Fig. 8. Fixed-ended beam.

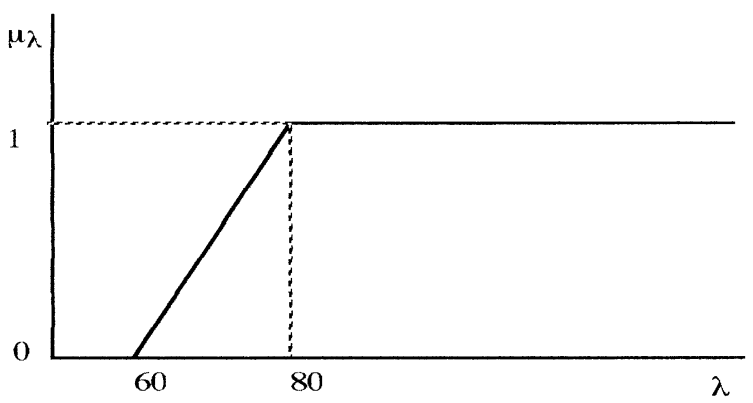

Fig. 9. Membership function for load.

may prefer the structure to resist a load $\lambda$ of 80 while he would accept a lower collapse load with diminishing support as indicated in Fig. 9.

The particular solution bending moment diagram and the complementarity solution diagrams are shown in Fig. 10.

According to MP3 the fuzzy optimization problem can be formulated as follows

$$
\begin{aligned}
& \text { Min } \quad 4 d \\
& \text { st : } d-p_{1} \geqslant 0 \\
& \quad d-p_{2} \geqslant 0 \\
& \quad d+0.25 p_{1}+0.75 p_{2} \geqslant 0.75^{*} 80[1-0.25(1-\alpha)] \\
& \quad \alpha \in[0 ; 1]
\end{aligned}
$$
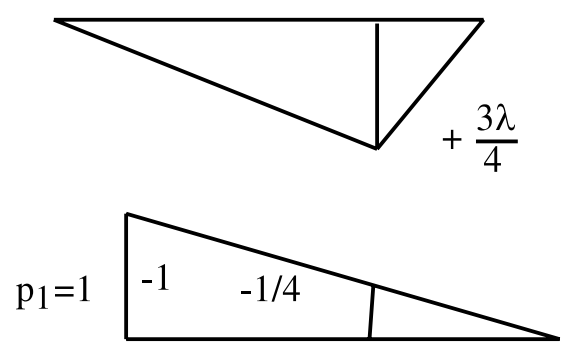

$\mathrm{b}_{1}$

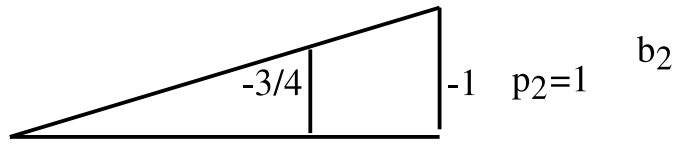

Fig. 10. Basic diagrams for fixed-ended beam. 
where $p_{1}$ and $p_{2}$ are the bending moments at the supports.

The first phase constraints in this problem are always in the $\alpha$ interval of variation so that:

$d=p_{1}=p_{2}=22.5+7.5 \alpha$

In the second phase the process to find the crisp solution using the bound search method is as follows:

$m=W[d(1)]=90 ; \quad M=W[d(0)]=120 ;$

$\mu_{G}(d)=0.75 \quad \alpha \in[0.75 ; 1] ; \quad \mu_{\mathrm{G}}(d)=3 /(3+\alpha)$

In this case there is an analytical expression for the fuzzy solution. The optimum level can be derived from Eq. (11) and the crisp solution is:

$\alpha^{*}=0.791 ; \quad p_{1}=p_{2}=d=28.43 ; \quad W\left[d\left(\alpha^{*}\right)\right]=113.73$

\subsection{Fuzzy material properties}

Alternatively if one considers uncertainty in the mechanical properties of the design variable $d$ represented in Fig. 11 together with the uncertainty of the loading, the fuzzy optimization problem becomes:

$\min 4 d$

$$
\begin{aligned}
\text { st }: & d[1+0.15(1-\alpha)]-p_{1} \geqslant 0 \\
& d[1+0.15(1-\alpha)]-p_{2} \geqslant 0 \\
& d[1+0.15(1-\alpha)]+0.25 p_{1}+0.75 p_{2} \\
& \geqslant 0.75 \times 80\left[1-0.25\left(1-\alpha^{*}\right)\right] \\
& \alpha \in[0 ; 1]
\end{aligned}
$$

Constraints are always critical when $\alpha \in[0,1]$ so that:

$d=p_{1}=p_{2}=\frac{22.5+7.5 \alpha}{1.15-0.15 \alpha}$

In the second phase bound search method leads to $\alpha \in[0.652,1]$ the crisp solution is

$\alpha^{*}=0.728 ; \quad p_{1}=p_{2}=d=26.87 ; \quad W\left[d\left(\alpha^{*}\right)\right]=107.47$

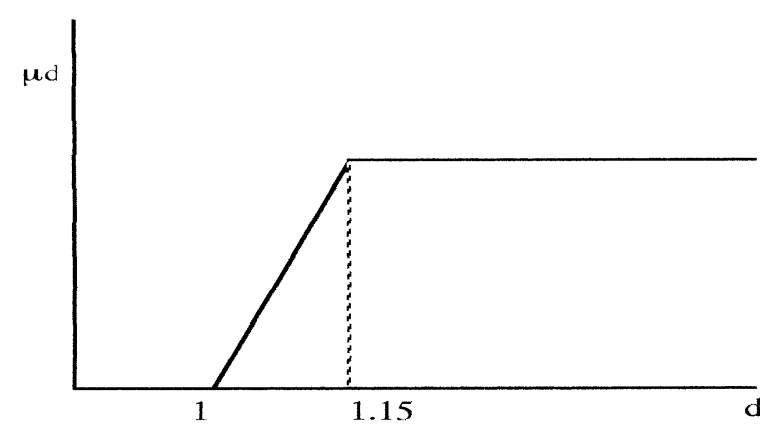

Fig. 11. Membership function for design variable $d$.

\subsection{Portal frame}

The three story three bay frame represented in Fig. 12 is designed to resist horizontal and vertical loads without collapsing plastically. The moment capacities of the beams and columns are the four design variables involved.

The particular solution and complementarity solution bending moments are shown in Fig. 13. If the loads are fuzzified as shown in Fig. 14, the first phase corresponds to solving the LP with the maximum and minimum $\alpha$ values:

\begin{tabular}{llllll}
\hline$\alpha$ & $d_{1}$ & $d_{2}$ & $d_{3}$ & $d_{4}$ & $W(\alpha)$ \\
\hline 0.0 & 0.324 & 0.865 & 0.135 & 0.622 & 10.757 \\
1.0 & 0.924 & 1.874 & 0.571 & 1.143 & 24.084 \\
\hline
\end{tabular}

In the general case the active set of constraints changes with $\alpha$. The limits of the $\alpha$ value are:

$m=10.7568 ; \quad M=24.0840 ; \quad \alpha \in[0.4466 ; 1]$

It can be seen from Table 1 that the crisp solution obtained by the bound search method is:

$\alpha^{*}=0.5813 ; \quad d_{1}=0.673 ; \quad d_{2}=1.452 ;$

$d_{3}=0.388 ; \quad d_{4}=0.925 ; \quad W^{*}=18.504$

\subsection{Reinforced concrete slab}

Consider the square slab with four edges clamped in Fig. 15 which is to be designed to resist the uniformly distributed load without collapsing plastically.

The top and bottom reinforcements will be allowed to be different, but within each slab region equal reinforcement is imposed a priori (Fig. 16). The symmetric features of the slab geometry and loading enables reinforcements in the $x$ and $y$ axes to be assumed equal hence only one eighth of the slab need be considered.

Both top and bottom reinforcement will have two design variables. The selected one eighth (ABC in Fig. 15)

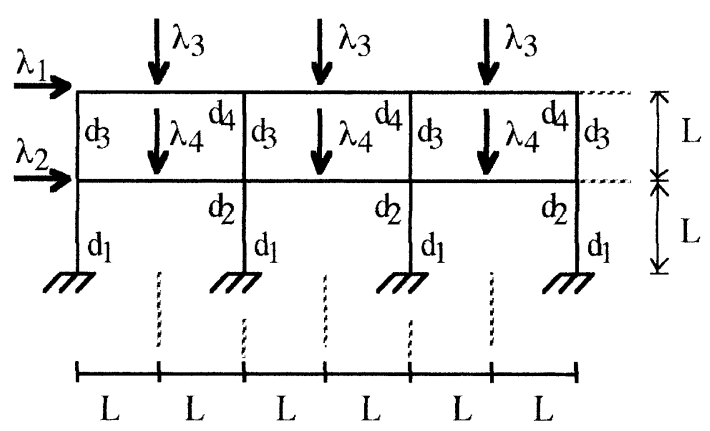

Fig. 12. Portal frame. 


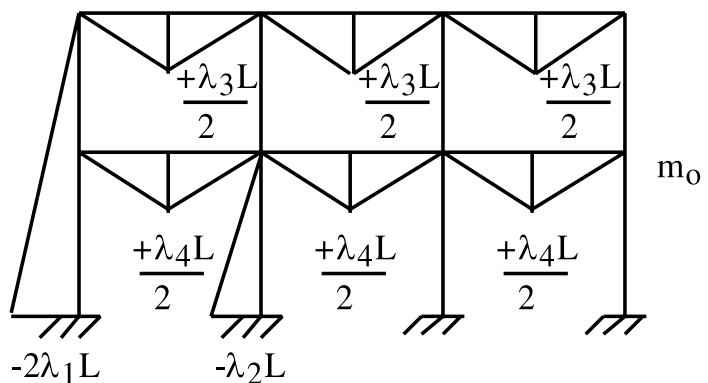

Fig. 13. Particular solution bending moments.
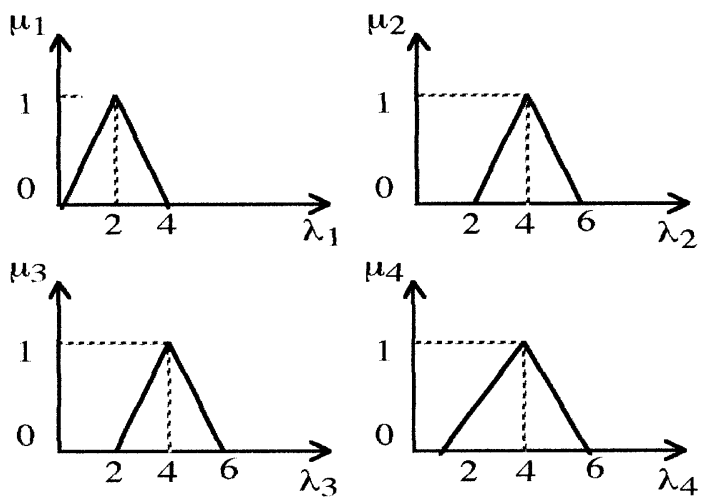

Fig. 14. Fuzzy loading.

Table 1

Bound search procedure

\begin{tabular}{llll}
\hline$k$ & $\alpha^{(k)}$ & $W\left(x^{k}\right)$ & $\mu \mathrm{G}^{(k)}$ \\
\hline 1 & 0.7233 & 20.396 & 0.5274 \\
2 & 0.6253 & 19.090 & 0.5635 \\
3 & 0.5943 & 18.677 & 0.5759 \\
4 & 0.5851 & 18.555 & 0.5797 \\
\hline
\end{tabular}

is discretized with four finite elements in such a way that each element will be located in only one design region so as to have a constant design variable, Fig. 17.

The constraint on deflection requires for a continuous span of $10 \mathrm{~m}$ and reinforcement (mild steel, $f_{y}=250$ $\mathrm{N} / \mathrm{mm}^{2}$ ) ratio of 0.5 , the total depth $h_{\mathrm{t}}$ of the slab to be at least $218.5 \mathrm{~mm}$. Here $h_{\mathrm{t}}=230 \mathrm{~mm}$ will be used.

With a characteristic live load $g_{k}=5 \mathrm{kN} / \mathrm{m}^{2}$ and unit weight of reinforced concrete $24 \mathrm{kN} / \mathrm{m}^{3}$, the ultimate load on the slab is $1.4 q_{k}+1.6 g_{k}=15.728 \mathrm{kN} / \mathrm{m}^{2}$. The nodal load vector $r_{0}$ can be obtained easily for the three free nodes as

$$
r_{0}^{\mathrm{t}}=\left[\begin{array}{lll}
16.3833 & 49.15 & 49.15
\end{array}\right]
$$

From the static equation (18), the transformation matrix $E$ is given by

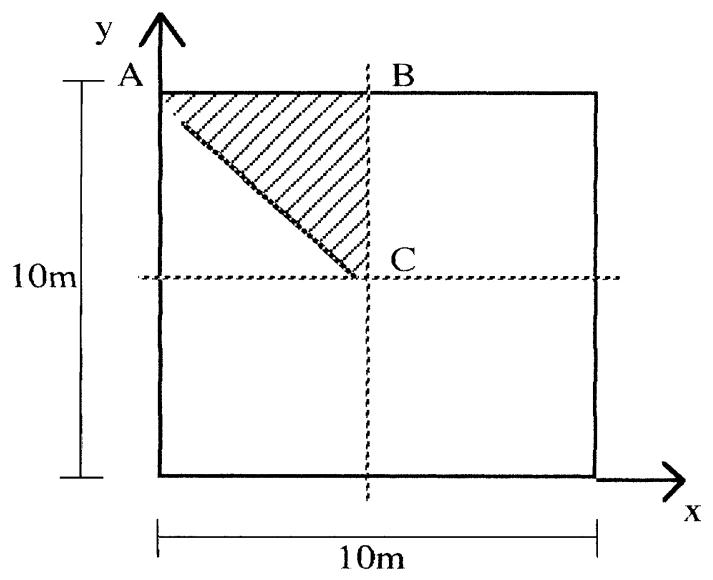

Fig. 15. Uniformly loaded square RC slab with four edges clamped.

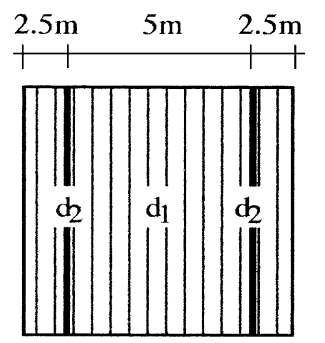

Bottom Reinforcement

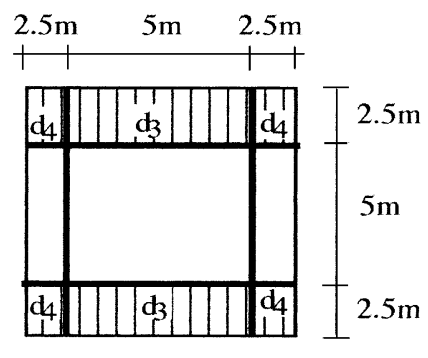

Top Reinforcement
Fig. 16. Arrangement of design variable $d_{i}$ in design regions identical for both $x$ and $y$ directions.

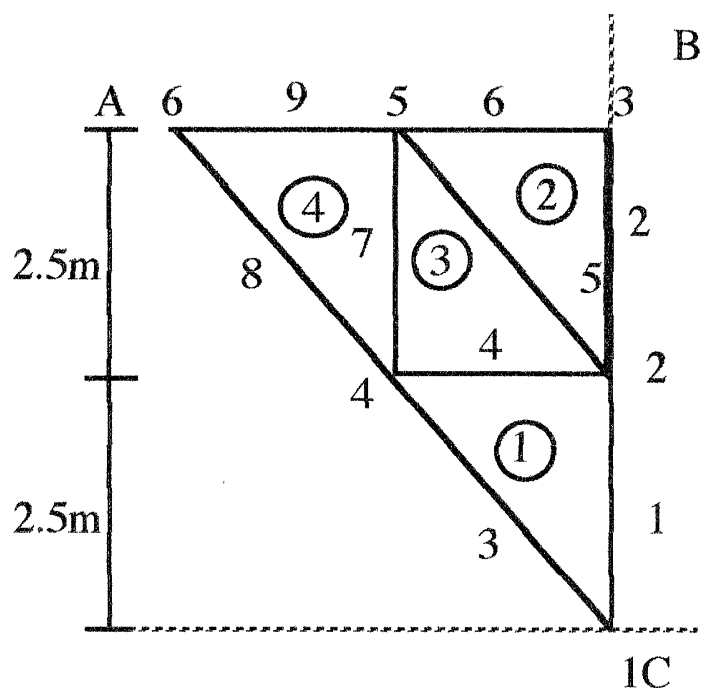

Fig. 17. Discretization of one-eighth $(\mathrm{ABC})$ of the square $\mathrm{RC}$ slab. 
$E=\left[\begin{array}{ccccccccc}0 & 0 & 0.2828 & -0.4 & 0 & 0 & 0 & 0 & 0 \\ 0.4 & 0 & -0.5657 & 0.4 & 0.2828 & -0.4 & -0.4 & 0 & 0 \\ -0.4 & 0 & 0.2828 & 0.4 & -0.5657 & 0 & 0.4 & 0.2828 & -0.4\end{array}\right]$

Matrix $J^{+}$(or $J^{-}$) relates the positive (or negative) plastic moment capacity of the four elements $m_{* 1}^{+}, m_{* 2}^{+}$, $m_{* 3}^{+}$and $m_{* 4}^{+}\left(\right.$or $m_{* 1}^{-}, m_{* 2}^{-}, m_{* 3}^{-}$and $m_{* 4}^{-}$) to the two design variables $d_{1}$ and $d_{2}$ (or $d_{3}$ and $d_{4}$ ).

Matrix $A_{1}$ (or $B_{1}$ ) relates plastic moment capacity $m$ across the interelement sides to the plastic moment capacity in the $x-(y)$ direction, $m_{x}^{*}$ (or $m_{y}^{*}$ ) of one of their connected elements whilst $A_{2}$ (or $B_{2}$ ) relates $m$ to $m_{x}^{*}$ (or $m_{y}^{*}$ ) of the elements on the other side. $A_{1}$ (or $A_{2}$ ) has elements of $l_{j} \sin ^{2} \theta_{j}$ and $B_{1}$ (or $B_{2}$ ) has $l_{j} \cos ^{2} \theta_{j}$.

$J^{+}=\left[\begin{array}{ll}1 & 0 \\ 0 & 1 \\ 0 & 1 \\ 0 & 1 \\ 1 & 0 \\ 1 & 0 \\ 1 & 0 \\ 0 & 1\end{array}\right]$

$$
J^{-}=\left[\begin{array}{ll}
0 & 0 \\
0 & 0 \\
0 & 0 \\
0 & 1 \\
0 & 0 \\
1 & 0 \\
1 & 0 \\
0 & 1
\end{array}\right]
$$

$A_{1}=\left[\begin{array}{cccc}2.5 & 0 & 0 & 0 \\ 0 & 2.5 & 0 & 0 \\ 1.7678 & 0 & 0 & 0 \\ 0 & 0 & 0 & 0 \\ 0 & 1.7678 & 0 & 0 \\ 0 & 0 & 0 & 0 \\ 0 & 0 & 2.5 & 0 \\ 0 & 0 & 0 & 1.7678 \\ 0 & 0 & 0 & 0\end{array}\right]$

$B_{1}=\left[\begin{array}{cccc}0 & 0 & 0 & 0 \\ 0 & 0 & 0 & 0 \\ 1.7678 & 0 & 0 & 0 \\ 2.5 & 0 & 0 & 0 \\ 0 & 1.7678 & 0 & 0 \\ 0 & 2.5 & 0 & 0 \\ 0 & 0 & 0 & 0 \\ 0 & 0 & 0 & 1.7678 \\ 0 & 0 & 0 & 2.5\end{array}\right]$

The sizes of the matrices $A_{2}$ and $B_{2}$ will be reduced by deleting those rows associated with boundary sides which are connected to the clamped edge.

$A_{2}=\left[\begin{array}{cccc}0 & 0 & 0 & 0 \\ 0 & 0 & 1.7678 & 0 \\ 0 & 0 & 0 & 0\end{array}\right] ;$
$B_{2}=\left[\begin{array}{cccc}0 & 0 & 2.5 & 0 \\ 0 & 0 & 1.7678 & 0 \\ 0 & 0 & 0 & 2.5\end{array}\right]$
$A \equiv\left[\begin{array}{c}A_{1} \\ - \\ A_{2}\end{array}\right] ; \quad B \equiv\left[\begin{array}{c}B_{1} \\ - \\ B_{2}\end{array}\right]$

Similar detection will be performed on the matrix $U$ for consistency.

$J^{+*} \equiv\left[\begin{array}{ll}A & B\end{array}\right] J^{+} \quad J^{-*} \equiv\left[\begin{array}{ll}A & B\end{array}\right] J^{-}$

$$
\begin{aligned}
& {\left[\begin{array}{cc}
2.5 & 0 \\
0 & 2.5 \\
3.5355 & 0 \\
2.5 & 0 \\
1.7678 & 1.7678 \\
2.5 & 0 \\
0 & 2.5 \\
0 & 3.5355 \\
0 & 2.5 \\
- & - \\
2.5 & 0 \\
1.7678 & 1.7678 \\
0 & 2.5
\end{array}\right]=\left[\begin{array}{cc}
0 & 0 \\
0 & 0 \\
0 & 0 \\
0 & 0 \\
1.7678 & 0 \\
2.5 & 0 \\
0 & 2.5 \\
0 & 3.5355 \\
0 & 2.5 \\
- & - \\
2.5 & 0 \\
1.7678 & 0 \\
0 & 2.5
\end{array}\right]} \\
& J \equiv\left[\begin{array}{cc}
J^{+*} & \bullet \\
\bullet & J^{-*}
\end{array}\right]
\end{aligned}
$$

If the thickness $h_{\mathrm{t}}$ of the slab is assumed uniform and if the lever arms $h_{x}^{+i}, h_{y}^{+i}, h_{x}^{-i}$ and $h_{y}^{-i}$ are assumed to be all equal to $h=0.85 h_{\mathrm{t}}$, the total reinforcement can be determined as

$v=0.85^{1} h_{\mathrm{t}} f_{y}\left[\begin{array}{llll}S^{\mathrm{T}} & S^{\mathrm{T}} & S^{\mathrm{T}} & S^{\mathrm{T}}\end{array}\right]\left[\begin{array}{l}m_{*_{x}}^{+} \\ m_{*_{y}}^{+} \\ m_{*_{*}}^{-} \\ m_{*_{y}}^{-}\end{array}\right]$

where $S^{\mathrm{T}}=\left[\begin{array}{llll}S_{1} & S_{2} & S_{3} & S_{4}\end{array}\right]$

Since the areas of the four elements are equal to $S=3.125 \mathrm{~m}^{2}$ and $f_{y}=2.5 \times 10^{5} \mathrm{kN} / \mathrm{m}^{2}$ is used, Eq. (21) becomes:

$v=6.39 \times 10^{-5}\left(e \bullet m^{*}\right)$

which can be expressed in terms of design variables $(d)$ as

$$
\begin{aligned}
v & =6.39 \times 10^{-5}(e \bullet J) d \\
& =1.2788 \times 10^{-4}\left[\begin{array}{llll}
2 & 2 & 1 & 1
\end{array}\right] d
\end{aligned}
$$

where $e$ is a row vector, all of whose elements are unity.

The objective function $z$ in LP (14) will be expressed as 
$z=\left[\begin{array}{llll}2 & 2 & 1 & 1\end{array}\right] d=V / \rho$

where $\rho=1.2788 \times 10^{-4}$

If the amount of main tensile reinforcement in a slab should be at least $0.25 \% b h_{\mathrm{t}}$. Hence

$\min A_{\mathrm{s}}=5.75 \times 10^{-4} \mathrm{~m}^{2}$ per m length

The equivalent plastic moment capacity can be evaluated as

$$
\begin{aligned}
\min m_{*} & =28.1031 \mathrm{kNm} / \mathrm{m} \\
& =d
\end{aligned}
$$

Now let the characteristic live load $g_{k}$ be the fuzzy quantity shown in Fig. 18.

The ULD on the slab will then be the fuzzy quantity in Fig. 19 and the vertical nodal forces can be shown to be the fuzzy quantities in Fig. 20. For simplicity, assume no fuzziness in the value of the lower bound of $\underline{d}$.

The additional matrices for the data are the vector for the softness of the nodal forces,

$\left(t_{r}^{1}\right)^{\mathrm{T}}=\left(t_{r}^{2}\right)^{\mathrm{T}}=\left[\begin{array}{lll}3.33 & 10 & 10\end{array}\right]$

and the vector for the nodal forces,

$\left(r_{0}\right)^{\mathrm{T}}=\left[\begin{array}{lll}16.383 & 49.15 & 49.15\end{array}\right]$

The optimal solutions with $\alpha=0$ and 1 are respectively:

$d_{1}^{*}=d_{2}^{*}=d_{3}^{*}=d_{4}^{*}=28.1031 \mathrm{kNm}$

$z^{*}=v^{*} / \rho=321.2503 \mathrm{~m}^{3}$

$d_{1}^{*}=d_{2}^{*}=d_{4}^{*}=28.1031 \mathrm{kNm}, \quad d_{3}^{*}=46.7697 \mathrm{kNm}$

$z^{*}=v^{*} / \rho=374.5703 \mathrm{~m}^{3}$

Therefore, the limits of the $\alpha$ value are $[0.858 ; 1]$ and the crisp solution obtained by the iterative bound search method described before is:

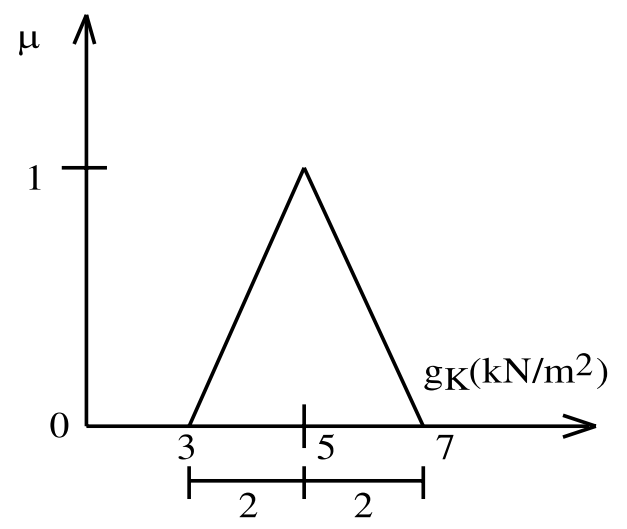

Fig. 18. Membership function of the characteristic live load.

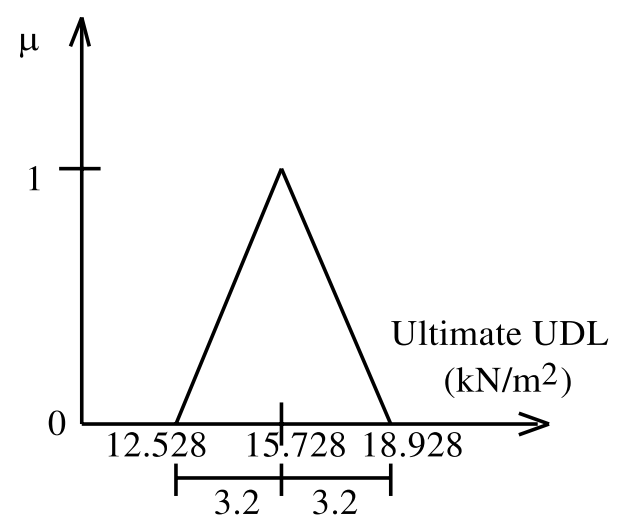

Fig. 19. Membership function of the ultimate UDL.

$$
\begin{aligned}
& \alpha^{*}=0.913 \quad d_{1}^{*}=d_{2}^{*}=d_{4}^{*}=28.1031 \mathrm{kNm}, \\
& d_{3}^{*}=44.423 \mathrm{kNm}, \quad z^{*}=v^{*} / \rho=369.877 \mathrm{~m}^{3}
\end{aligned}
$$

It will be noticed the same trends displayed by the frame examples are demonstrated in this $\mathrm{RC}$ slab

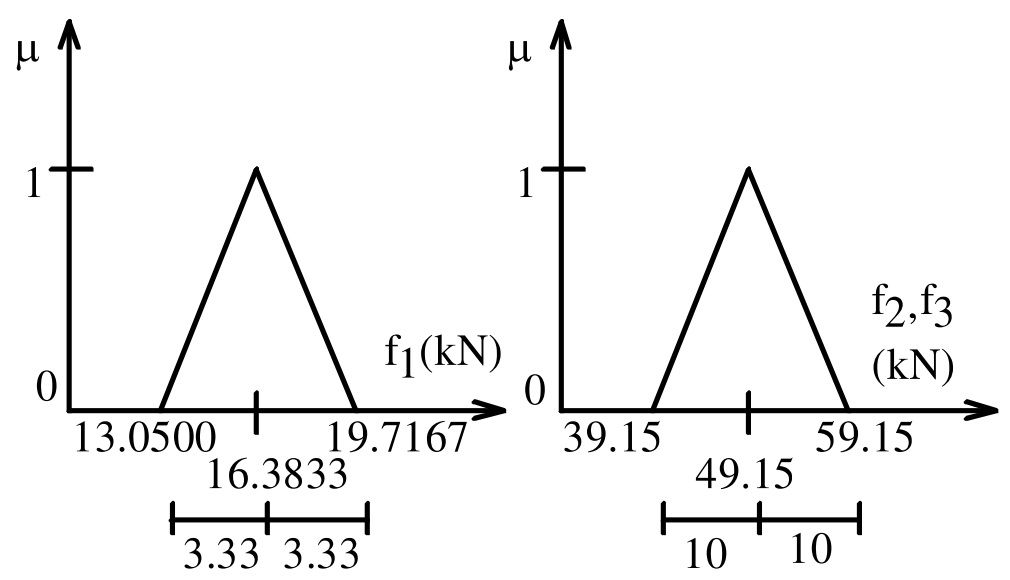

Fig. 20. Membership function of the applied nodal forces on the discretized slab. 
example. The optimal weight for the deterministic calculation (374.5703) was reduced to a smaller number (369.877) for the first fuzzy calculation which corresponds to an acceptability measure of 0.913 .

\section{Conclusions}

It is pointed out that fuzzy optimization has the advantage of ordinary optimization with a more realistic model of fuzzy constraints taken into account. Hence the structural design should be more reasonable and beneficial. The illustrative numerical examples given here show that the two-phase method based on fuzzy set theory seems to be a rational and effective approach for the fuzzy optimization of structures with plastic or elastic material behavior. The fuzzy solution is obtained in the first phase in accordance with the Ralescu point of view that a fuzzy problem should have a fuzzy solution [9]. The crisp solution which maximizes the membership function of fuzzy decision-making can then be found from the fuzzy solution in the second phase. As a result, the proposed approach provides favorable condition for selection of structural design schemes so as to have a higher $\alpha$-level and to save materials.

\section{References}

[1] Simões LMC. Reliability-based plastic synthesis of portal frames. Eng Opt 1991;17:175-204.

[2] Simões LMC. Reliability-based plastic synthesis of reinforced concrete slabs. In: S. Hernandez, C.A. Brebbia editors. Computer aided optimum design of structures: applications. Computational Mechanics Publications, 1997. p. 163-72.

[3] Barbieri E, Cinquini C, Lombardi M. Shape/size optimization of truss structures using non-probabilistic description of uncertainty. In: C.A. Brebbia, S. Hernandez editors. Computer aided optimum design of structures Springer, 1989. p. 285-95.

[4] Zadeh LA. Fuzzy sets. Inf Control 1965;8:338-53.

[5] Wang G-Y, Wang W-Q. Fuzzy optimum design of structures. Eng Opt 1985;8:291-300.

[6] Chuang P-H. Fuzzy mathematical programming in civil engineering systems, PhD Thesis. Imperial College of Science and Technology, University of London, 1985.

[7] Bellman RE, Zadeh LA. Decision-making in a fuzzy environment. Mgmt Sci 1970;17:151-69.

[8] Verdegay JL. Fuzzy mathematical programming. In: M.M. Gupta, E. Sanchez, editors. Fuzzy information and decision processes. New York: North-Holland; 1982. p. 231-37.

[9] Ralescu D. Inexact solutions for large scale control problems. Proc. Int. 1st Cong Math Service of Man, Spain, 1977. 